\title{
In-situ Switching of a Ferroelectric Film Through a Non-ferroelectric Layer and Direct Scanning Probe Analysis of the Same Cross Section
}

\author{
J.R. Jokisaari ${ }^{1}$, P. Gao ${ }^{1}$, and X.Q. Pan $^{1}$ \\ ${ }^{1}$ Department of Materials Science and Engineering, University of Michigan, Ann Arbor, MI 48109
}

Ferroelectric oxides are of continuing interest in a new generation of electronic devices, such as piezoelectric actuators and nonvolatile memory devices. In general, their application involves manipulation of the polarization of the ferroelectric and the polarization depends sensitively on boundaries and interfaces. Here we study the dynamic interaction during polarization switching of domains with a thin STO layer of a complex film stack composed of ferroelectric, followed by a very thin non-ferroelectric STO layer, and a second ferroelectric layer.

In-situ transmission electron microscopy (TEM) is a unique tool allowing direct examination of domain dynamics in real time in ferroelectric materials and coupled with high-resolution aberration corrected imaging, atomic-scale structure. Additionally, assorted scanning probe methods including piezoresponse force microscopy (PFM) conductive AFM, and kelvin probe AFM can be used to characterize the domain structure and electronic properties, though these methods are strictly confined to the surface of a sample. These SPM methods compliment TEM structural and electronic data, allowing direct measurement of the electronic properties of the same specimen including electrical conductivity, leakage currents, and surface potentials.Here, in-situ TEM and high-resolution aberration-corrected STEM, combined with PFM, CAFM, and KFM were used to examine the evolution of polarization domain structure with respect to the STO interlayer on BFO and PZT thin films. (001) oriented BFO thin films with the structure BFO/STO/BFO/LSMO grown by molecular beam epitaxy or $\mathrm{PZT} / \mathrm{STO} / \mathrm{PZT} / \mathrm{SCRO}$ grown by PLD on orthoscandate substrates. Cross sections were prepared by mechanical polishing followed by argon ion-milling. TEM specimens were used for SPM as well as electron imaging. Films were characterized by JEOL 2010F, 3011, and 2100F and the TEAM0.5 FEI titan (TEM), and the NT-MDT Spectra SPM. In-situ TEM was carried out using a specialized holder from Nanofactory Instruments.

The STO layer is very thin, but it has a very strong effect on polarization switching in these films. In-situ switching of both BFO/STO/BFO/LSMO/TSO and PZT/STO/PZT/SCRO/DSO films is shown in Fig. 1. Both ferroelectrics show similar behavior, with classing nucleation and growth of the domain from the top surface, while the bottom film layer switches much more rapidly and to a much greater extent under the same bias. This is most visible in the PZT sample, where a single domain grows downward from the tip contact and terminates at the STO interlayer, while multiple nuclei grow from the bottom surface to the STO layer but do not penetrate to the surface. When the voltage was released, the switched domain disappeared. Without bottom electrode, the quality of BFO thin film has been improved significantly with clear period domain patterns and much less defects, as shown in Fig. 2 , which is a dark-field TEM image viewed from the [110] direction. The second panel gives a high-resolution image of the STO layer in the BFO. SPM is employed to study the electronic properties underlying the switching behavior in this film structure, Figure 3 shows the sample configuration for SPM studies, where a TEM specimen was coated from the back side with an Au electrode allowing through-specimen current measurements to be carried out. This has the advantage of direct correlation with the TEM results, as the sample has already been observed in TEM, while allowing the full array of surface probe measurements available in SPM. For example, CAFM shows the film to be slightly 
conductive, but the STO layer is .not. However, it does appear in the PFM image, indicating that, in this case, the STO directly affects the electrical boundary condition, producing the observed anomalous switching behavior

In conclusion, the combination of TEM and SPM techniques on the same samples allows direct access to both detailed structure and electronic property measurements. This combination of techniques allows determination of the mechanism underlying the unusual switching behavior intehigh electriedifigation of the internal electric field by the STO interlayer.
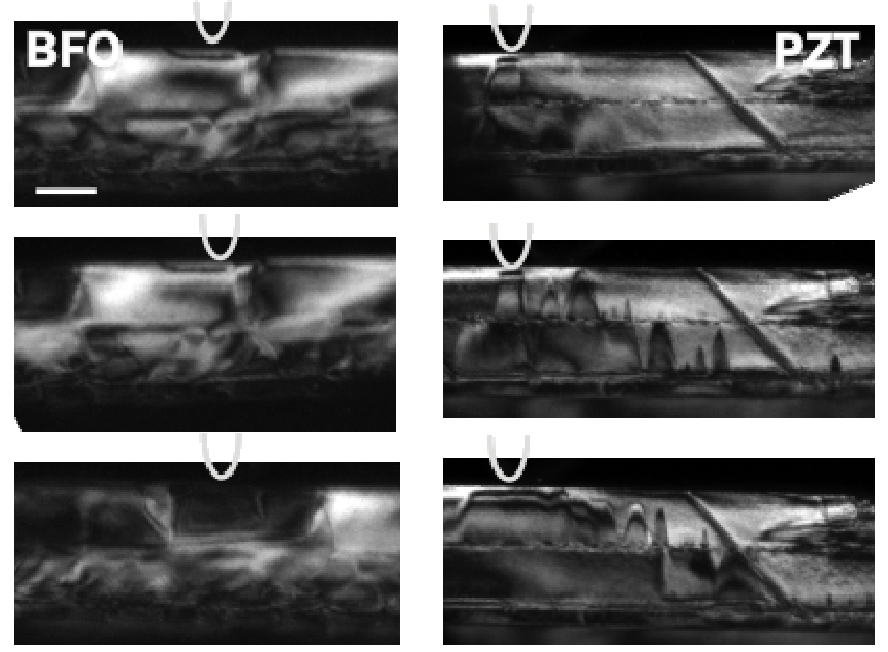

Fig. 1 Dark field images taken in-situ with a voltage applied to the sample. The BFO sample on the left is more defective and switching is less clear than in the PZT to the right. The scale bar is $50 \mathrm{~nm}$.
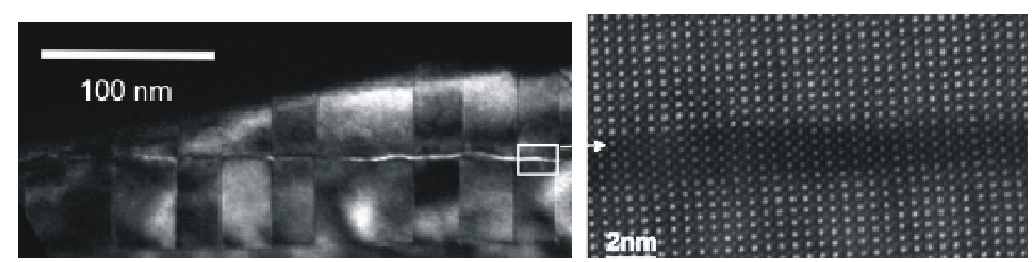

Fig. 2 Dark field image showing the BFO film stack and domain structure, without the LSMO back electrode. This sample was used for the SPM study below.
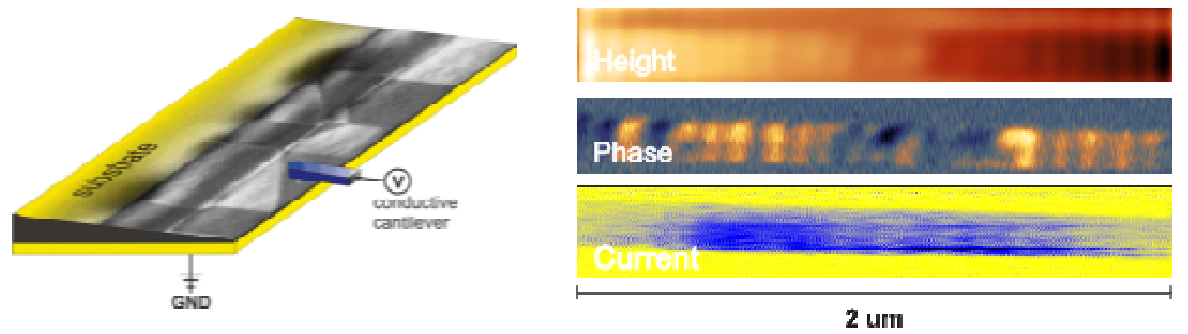

Fig. 3 The left panel shows a schematic of the SPM sample. A gold electrode was sputtered on the back side of the TEM specimen to look at the conduction through the film. The panel on the right shows the height, PFM phase, and CAFM current measurement captured on consecutive scans. 